\title{
Single-Layer Versus Multilayer Preplanned Lightpath Restoration
}

\author{
Alessio Giorgetti, Luca Valcarenghi, Member, IEEE, and Piero Castoldi, Associate Member, IEEE
}

\begin{abstract}
Upon failure occurrence, preplanned lightpath restoration recovers a faulted lightpath by selecting the backup path from a set of preplanned paths. Preplanned lightpath restoration combines the fast recovery time guaranteed by off-line backup path computation with the adaptive recovery guaranteed by the on-line choice of the backup path. One of the main issues in preplanned lightpath restoration is the backup path selection. To address this problem, stochastic preplanned restoration schemes have been proposed. In this study, a family of deterministic preplanned restoration schemes is introduced and evaluated. The deterministic preplanned restoration with proportional weighted path choice (DPR-PW) and the multilayer DPR-PW (MDPR-PW) schemes are proposed as single-layer (e.g., optical layer) and multilayer (e.g., sublightpath granularity) preplanned restoration schemes, respectively. Both schemes utilize the same network state information employed in stochastic preplanned restoration schemes. The originality of both DPR-PW and MDPR-PW is the deterministic choice of the paths for recovering disrupted lightpaths. The choice minimizes the error between the fraction of lightpaths assigned to each backup path and the backup path selection probabilities that, in turn, are computed to minimize resource contention, as in the stochastic schemes. Numerical results show that, if lightpath capacity is fully utilized, then DPR-PW, MDPR-PW, and multilayer stochastic preplanned restoration schemes achieve similar performance in terms of fraction of recovered connections. However, while both deterministic and stochastic multilayer schemes do require network nodes to implement sublightpath connection grooming capabilities, the DPR-PW scheme does not. Thus, DPR-PW optimizes recovery performance by slightly increasing the intranode coordination required during lightpath recovery with respect to stochastic schemes but without impacting the overall network cost. Finally, in all the considered schemes, the aggregation of the signaling necessary for reserving and activating spare bandwidth along backup paths allows to limit the signaling overhead while negligibly increasing the recovery time.
\end{abstract}

Index Terms-Deterministic, lightpath, multilayer, next generation SONET/SDH, preplanned restoration, single layer, stochastic, wavelength routed networks, WDM.

\section{INTRODUCTION}

$\mathbf{R}$ ESTORATION schemes are emerging as a main candidate for providing dynamic all-optical end-to-end connections (i.e., lightpaths) with the capability of overcoming

Manuscript received December 16, 2004; revised July 12, 2005. This work was supported by the Italian Ministry of University and Research (MIUR) in part under the INTREPIDO PRIN project and in part under the FIRB project "Enabling platforms for high-performance computational grids oriented to scalable virtual organizations (GRID.IT)."

The authors are with the Center of Excellence for Communications Network Engineering (CEIRC), Scuola Superiore Sant'Anna di Studi Universitari e di Perfezionamento, 56124 Pisa (PI), Italy (e-mail: agiorgetti@sssup.it; valcarenghi@sssup.it; castoldi@sssup.it).

Digital Object Identifier 10.1109/JLT.2005.856156 network failures. On one hand, restoration schemes provide recovery time ${ }^{1}$ longer than the one guaranteed by shared and dedicated protection schemes that allocate resources along both the working and the backup path upon connection arrival [1], [2]. On the other hand, restoration schemes have the potential of utilizing network spare resources more efficiently and thus recovering a higher fraction of connections. Indeed, by finding backup paths just upon failure occurrence (i.e., on-line), restoration schemes can resort to more up-to-date network state information. Moreover, restoration schemes can naturally handle simultaneous multiple failures [3]. For instance, restoration schemes are capable of recovering a high fraction of connections disrupted by simultaneous multiple failures even if spare capacity for recovering all the connections from just a single failure is provided [4].

Preplanned restoration schemes have been proposed [5], [6] to improve restoration scheme recovery time while maintaining the advantage of on-line backup path selection [4]. In preplanned restoration, a set of backup paths along which to reroute failed connections is precomputed. Upon failure occurrence, just one of the preplanned backup paths is selected to recover a disrupted connection. The backup path selection represents the preplanned restoration scheme main issue. If the network is dimensioned for recovering all the connections from all the considered failure scenarios, the optimal backup path selection can be obtained by solving the integer linear programming formulation of the path restoration routing (PRR) problem [7]. The PRR problem consists of the multicommodity maximum flow (MCMF) problem of assigning failed connections to a set of precomputed backup paths. Because the PRR problem is an nondeterministic polynomial (NP)-hard problem, its on-line solution is quite impractical [7].

Backup path selection methods based on heuristics have been proposed to approximate the optimal solution of both the PRR problem in networks capable of fully recovering all the failed connections from the failure and the PRR problem adaptation to predimensioned fixed-capacity networks not able to assure recovery to all the failed connections [6]-[9]. During the backup path selection, the proposed heuristics consider the potential contention for network spare resources. In particular, in the stochastic preplanned restoration with proportional weighted path choice (SPR-PW) scheme [6], [8], the backup path selection is based on probabilities that, in turn, depend on the network state information available at the nodes upon failure

\footnotetext{
${ }^{1}$ In this study, recovery time is defined as the time elapsed from the instant in which the failure is notified at the connection source node to the instant in which the transmission is resumed.
} 
occurrence and on the forecast network link occupation after lightpath recovery. The multilayer SPR-PW (MSPR-PW) scheme [9] combines the concept supported by the generalized multiprotocol label switching (GMPLS) framework of hierarchical label switched paths (LSPs) [10], [11] and the backup path stochastic selection proper of the SPR-PW scheme. By applying the backup path stochastic selection to lower-order LSPs [e.g., gigabit Ethernet (GbE), asynchronous transfer mode (ATM), synchronous optical network/synchronous digital hierarchy (SONET/SDH) connections] carried by the failed lightpaths in a multilayer network architecture, MSPR-PW is able to increase the fraction of recovered connections with respect to the SPR-PW scheme. However, MSPR-PW best performance is obtained in the ideal situation in which all the established lower-order LSPs are characterized by the same bandwidth and all the network nodes feature connection (de)multiplexing (i.e., grooming) capabilities.

In this paper, a family of deterministic preplanned restoration schemes is proposed and evaluated. The proposed deterministic schemes utilize the same network state information available in the stochastic preplanned restoration schemes such as SPR-PW and MSPR-PW. The novelty of the proposed deterministic schemes is their backup path selection method. The selection is deterministically made with the objective of minimizing the square Euclidean distance between the fraction of lightpaths assigned to a backup path and the backup path selection probabilities. As in the stochastic preplanned restoration schemes, the backup path selection probabilities are computed based on the network state information and the forecast contention for spare resources during connection recovery. Given the available network state information, the backup path selection based on the computed probabilities maximizes the likelihood of recovering failed lightpaths. The deterministic preplanned restoration with proportional weighted path choice scheme (the DPR-PW scheme) is proposed as the single-layer (e.g., optical layer) restoration scheme, while the multilayer DPR-PW (the MDPR-PW scheme) is proposed as the multilayer (e.g., sublightpath granularity) restoration scheme. The DPR-PW scheme aims at maximizing the fraction of recovered lightpaths by optimizing the consistency between the performed backup path selection and the optimal backup path selection probabilities. In addition, the MDPR-PW scheme exploits the possibility of recovering disrupted lightpaths at the sublightpath granularity; in return, it requires additional node grooming capabilities.

The proposed DPR-PW and MDPR-PW schemes are compared with the SPR-PW scheme, the MSPR-PW scheme, and the optimal solution of the PRR problem adaptation to fixedcapacity networks. Three different network scenarios are considered where grooming capabilities are available: 1) at each network node (i.e., multihop grooming); 2) at just the connection source and destination nodes (i.e., single-hop grooming); and 3) at no nodes (i.e., no grooming) [12]. Numerical results show that the DPR-PW scheme outperforms the SPR-PW performance in terms of restoration blocking probability. ${ }^{2}$ In

\footnotetext{
${ }^{2}$ In this paper, restoration blocking probability is defined as the ratio between the total bandwidth of unrestored connections and the total bandwidth of failed connections.
}

addition, the DPR-PW scheme guarantees restoration blocking probabilities lower than or similar to the ones guaranteed by multilayer stochastic and deterministic preplanned restoration schemes but does not require interlayer coordination. On the other hand, with respect to the SPR-PW scheme, the DPR-PW scheme exhibits a higher recovery time because it requires coordination in the recovery attempts of connections between the same node pair. However, the amount of signaling required by the DPR-PW scheme during lightpath recovery is similar to the one required by the SPR-PW scheme.

\section{InVESTIGATED RESTORATION SCHEMES}

In this section, the network model and the parameters utilized by the investigated schemes are presented. The single-layer DPR-PW scheme is introduced and two possible implementations are proposed. Then, the MSPR-PW and MDPR-PW schemes are detailed.

\section{A. Network Model}

The schemes proposed in this study are applied to the restoration of lightpaths, i.e., end-to-end all-optical connections, disrupted by a single physical bidirectional link failure such as fiber link disruption. The following parameters, partially introduced in [6], are utilized in all the considered schemes:

- $G(\mathcal{N}, \mathcal{L})$ is the graph modeling the physical network with a set of $|\mathcal{N}|$ nodes and $|\mathcal{L}|$ bidirectional links;

- $\mathcal{W}_{s, d}=\left\{v_{s, d}^{1}, \ldots, v_{s, d}^{i}, \ldots, v_{s, d}^{q}\right\}$ is the set of $q$ working lightpaths established between source node $s$ and destination node $d$;

- $\mathcal{R}_{s, d}=\left\{r_{s, d}^{1}, \ldots, r_{s, d}^{i}, \ldots, r_{s, d}^{k}\right\}$ is the set of $k$ precomputed (i.e., preplanned) backup paths between the node pair $(s, d)$;

- $\mathcal{L} \mathcal{S} \mathcal{P}_{s, d}^{i}=\left\{L S P_{s, d}^{i, 1}, \ldots, L S P_{s, d}^{i, j}, \ldots, L S P_{s, d}^{i, n}\right\}$ is the set of $n$ lower-order LSPs multiplexed in the working lightpath $v_{s, d}^{i}$;

- $c_{l}$ is the total capacity of link $l \in \mathcal{L}$

- $\mu_{l}$ is the utilized working capacity along link $l \in \mathcal{L}$;

- $\lambda_{l}^{\bar{l}}$ is the potential capacity required on $\operatorname{link} l \in \mathcal{L}$ to restore lightpaths disrupted by a failure on link $\bar{l} \in \mathcal{L}$.

For any $(s, d)$ pair, all the working lightpaths belonging to the set $\mathcal{W}_{s, d}$ are routed along the shortest path between node $s$ and node $d$. The backup path set $\mathcal{R}_{s, d}$ consists of the $k$ paths link disjoint from the working path and maximally link disjoint between themselves. The link disjointness of the backup paths from the working path is obtained by removing the working path edges from the graph $G(\mathcal{N}, \mathcal{L})$ during backup path computation. Maximal link disjointness between the backup paths is obtained by increasing the cost of the graph $G(\mathcal{N}, \mathcal{L})$ edges spanned by the computed backup path after each backup path computation. The backup path maximal link disjointness improves restoration scheme robustness against multiple failures because it maximizes the number of backup paths surviving a failure.

The three parameters $c_{l}, \mu_{l}$, and $\lambda_{l}^{\bar{l}}$ are expressed in terms of number of wavelengths. Network nodes store them for each $l$ and $\bar{l}$ in a local database that is periodically updated to keep the 
values consistent with the current network state. During the provisioning phase, signaling messages based on constraint-based routing label distribution protocol (CR-LDP) or resource reservation protocol with traffic engineering extensions (RSVP-TE) [8], [11] are sent along working paths to reserve bandwidth resources and along backup paths in order to update node databases without reserving spare resources. This information is subsequently advertised to all the network nodes by using the open shortest path first (OSPF) routing protocol extensions proposed for GMPLS networks [8], [10], [11].

As introduced in [6], based on $c_{l}, \mu_{l}$, and $\lambda_{l}^{\bar{l}}$, each source node $s$ computes the weight $w_{r_{s, d}^{i}}^{\bar{l}}$ for each destination $d$, for each backup path $r_{s, d}^{i}$, and for each link failure scenario $\bar{l}$, i.e.,

$$
w_{r_{s, d}^{i}}^{\bar{l}}=\min _{l \in r_{s, d}^{i}}\left(\frac{c_{l}-\mu_{l}}{\lambda_{l}^{\bar{l}}}\right) .
$$

The path selection probability $P_{r_{s, d}^{i}}^{\bar{l}}$ associated to each backup path is then computed as

$$
P_{r_{s, d}^{i}}^{\bar{l}}=\frac{w_{r_{s, d}^{i}}^{\bar{l}}}{\sum_{i=1}^{k} w_{r_{s, d}^{i}}^{\bar{l}}} .
$$

\section{B. Overcoming Multiple Failures}

Single link failures are the predominant form of failures in optical networks [13]. However, the utilization of $k$ maximally link disjoint preplanned backup paths allows the considered preplanned restoration schemes to potentially overcome multiple failures. For instance, if $k$ link disjoint backup paths are preplanned, at least $k-1$ backup paths result available for recovering a disrupted lightpath from any dual failure scenario. To adapt the proposed preplanned restoration to a dual failure scenario, the substitution of the parameter $\lambda_{l}^{\bar{l}}$ with a parameter $\lambda_{l}^{\bar{l}, \bar{m}}$ is required. The parameter $\lambda_{l}^{\bar{l}, \bar{m}}$ represents the potential capacity utilized on link $l$ for recovering lightpaths disrupted by a dual failure on link $\bar{l}$ and $\bar{m} \in \mathcal{L}$. However, a suboptimal backup path selection can still be performed by utilizing the backup path selection probabilities computed through $\lambda_{l}^{l}$. The suboptimal selection is based on excluding backup paths affected by the second failure (i.e., by assigning to them a selection probability equal to zero) and renormalizing the remaining backup path choice probabilities.

\section{DPR-PW Scheme}

The DPR-PW scheme is a single-layer (e.g., optical layer) preplanned restoration scheme in which backup paths are deterministically selected with the objective of minimizing the square Euclidean distance between the fraction of lightpaths assigned to backup paths and the backup path selection probabilities $P_{r_{s, d}^{i}}^{\bar{l}}$. The backup path selection probabilities are the ones utilized in SPR-PW. Given their computation method and the available network state information, they represent the fraction of disrupted lightpaths assigned to a backup path that minimizes the contention for network spare resources. Thus, the DPR-PW scheme utilizes the same network state information employed in the SPR-PW scheme [8] but aims at improving the SPR-PW restoration blocking probability by optimizing the backup path selection strategy without requiring additional signaling or node capabilities such as grooming capabilities.

1) DPR-PW Description: In the DPR-PW scheme, upon failure occurrence, each node $s$ identifies the lightpaths, originating from itself, that are disrupted by the failure. Then, for each destination $d$, it assigns each disrupted lightpath, connecting the same node pair $(s, d)$, to one of the $k$ preplanned backup paths belonging to the set $\mathcal{R}_{s, d}$. For each destination $d$, the backup path selection provides $k$ subsets of lightpaths. Each subset contains the lightpaths assigned to the same backup path.

The assignment of the disrupted lightpaths to the preplanned backup paths is formally defined by utilizing the following sets:

1) $\Pi^{\bar{l}}(s, d)$ is the set of $|s, d|^{\bar{l}}$ lightpaths between the node pair $(s, d)$ disrupted by the link $\bar{l}$ failure;

2) $\Pi_{i}^{\bar{l}}(s, d)$ is the set of $|s, d|_{i}^{\bar{l}}$ disrupted lightpaths between the node pair $(s, d)$ assigned to backup path $r_{s, d}^{i}$ upon link $\bar{l}$ failure.

Given the failure of link $\bar{l}$, the lightpath subsets $\Pi_{i}^{\bar{l}}(s, d)$ are formed so that all the disrupted lightpaths between the same node pair $(s, d)$ are assigned to one and only one backup path

$$
\sum_{i=1}^{k}|s, d|_{i}^{\bar{l}}=|s, d|^{\bar{l}} \quad \forall \bar{l}
$$

where $|s, d|_{i}^{\bar{l}} \geq 0$. The square Euclidean distance $D_{S, P}$ between the fraction of lightpaths assigned to a backup path and the backup path selection probabilities $P_{r_{s, d}^{i}}^{\bar{l}}$ is defined as the sum, for each backup path, of the squared difference between the ratio of lightpaths assigned to the backup path and the number of disrupted lightpaths and the backup path selection probability

$$
D_{S, P}=\sum_{i=1}^{k}\left(\frac{|s, d|_{i}^{\bar{l}}}{|s, d|^{\bar{l}}}-P_{r_{s, d}^{i}}^{\bar{l}}\right)^{2} .
$$

The objective of the DPR-PW scheme is to minimize $D_{S, P}$ so that the consistency between the backup path assignment and the backup path selection probability is maximized.

2) DPR-PW Algorithm Implementations: Two possible implementations of the DPR-PW scheme are proposed. The two implementations differ in the procedure utilized for building the lightpath subsets.

The exhaustive DPR-PW implementation, namely E-DPR$\mathrm{PW}$, utilizes an exhaustive search to choose the lightpath assignment that minimizes $D_{S, P}$. In the E-DPR-PW implementation, upon link failure notification, for each destination node $d$ each source node builds a table containing a number of rows equal to the possible $\left(\begin{array}{c}|s, d|^{l}+k-1 \\ k-1\end{array}\right)$ assignments of the $|s, d|^{\bar{l}}$ disrupted lightpaths to the $k$ preplanned backup paths. Each row also contains the $D_{S, P}$ value correspondent to each assignment. Based on the table, the source node selects the row that minimizes $D_{S, P}$. After the selection of the optimal backup path assignment, the source node starts the restoration attempt along the selected backup paths. 
The iterative DPR-PW implementation, namely I-DPR-PW, utilizes an iterative greedy assignment of disrupted lightpaths to the subsets. Assuming that a set of $N$ lightpaths between the same $(s, d)$ pair is disrupted by the failure of link $\bar{l}$ (i.e., $|s, d|^{\bar{l}}=N$ ) at a specific iteration $n$ of the overall $N$ iterations, the source node $s$ assigns one disrupted lightpath to a backup path so that the distance $D_{S, P}^{n}$, computed after $n$ iterations, is minimal. Specifically

$$
D_{S, P}^{n}=\min _{j \in\{1, \ldots, k\}} \sum_{i=1}^{k}\left(\frac{|s, d|_{i}^{n-1, \bar{l}}+\delta_{j}}{n}-P_{r_{s, d}^{i}}^{\bar{l}}\right)^{2}
$$

where $\delta_{j}$ is a binary variable that is equal to 1 if $i=j$ and 0 otherwise, and $|s, d|_{i}^{n-1, \bar{l}}$ is the number of lightpaths assigned to subset $i$ until iteration $n-1$.

In this scenario, the constraint in (3) becomes

$$
\sum_{i=1}^{k}|s, d|_{i}^{n, \bar{l}}=n
$$

for each iteration $n$ and the specific link $\bar{l}$ failure.

The computational complexity of the two proposed DPR-PW scheme implementations can be measured in terms of the number of Euclidean distances to be computed by the source node $s$ to assign all the disrupted lightpaths between the $(s, d)$ pair to the preplanned backup paths. In particular, the E-DPR-PW implementation requires the computation of $\left(\begin{array}{c|c|c|}\mid s, k-1 \\ k-1\end{array}\right)$ distances, while I-DPR-PW needs $k \times|s, d|^{\bar{l}}$ distance computations (i.e., $k$ distance computations for each iteration). Therefore, I-DPR-PW presents a significantly lower computational complexity than E-DPR-PW.

On the other hand, while the E-DPR-PW implementation guarantees the selection of the optimal backup path assignment, the I-DPR-PW implementation might provide a suboptimal assignment because of its greedy nature. Nevertheless, the optimality of the I-DPR-PW implementation, when two preplanned backup paths are available, can be proved (Appendix).

3) DPR-PW Example: The effectiveness of the DPR-PW scheme with respect to the SPR-PW scheme is explained by the example depicted in Fig. 1. Network links and lightpaths are assumed to be bidirectional and two $(k=2)$ preplanned backup paths are computed for each established lightpath. Suppose that each network link has a capacity of ten wavelengths. Two lightpaths ${ }^{3}$ are established between node pair $(0,5)$, nine lightpaths are established between node pair $(2,3)$, and one lightpath is established between node pair $(1,5)$ and node pair $(1,4)$. The network utilization parameters are as follows:

- $c_{l}=10 \forall l$

- $\mathcal{W}_{0,5}=\left\{v_{0,5}^{1}, v_{0,5}^{2}\right\},\left|\mathcal{W}_{0,5}\right|=2$

- $\mathcal{W}_{2,3}=\left\{v_{2,3}^{1}, \ldots, v_{2,3}^{9}\right\},\left|\mathcal{W}_{2,3}\right|=9$

- $\mathcal{W}_{1,5}=\left\{v_{1,5}^{1}\right\},\left|\mathcal{W}_{1,5}\right|=1$;

- $\mathcal{W}_{1,4}=\left\{v_{1,4}^{1}\right\},\left|\mathcal{W}_{1,4}\right|=1$;

- $\mu_{(0,1)}=1, \mu_{(0,5)}=3, \mu_{(1,4)}=1, \mu_{(2,3)}=9$;

- $\mu_{l}=0$ for the other links.

${ }^{3}$ For clarity, in Fig. 1, only one working lightpath with $k=2$ backup paths is depicted for each $(s, d)$ pair.

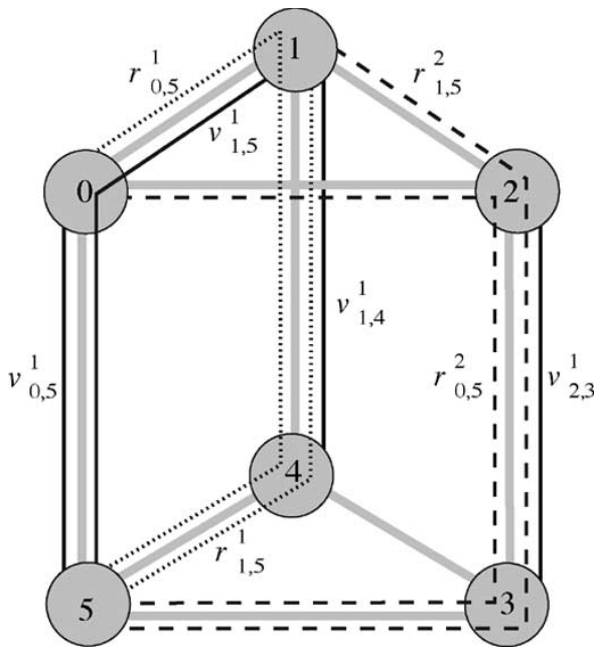

Fig. 1. Example of failure restoration in the test network topology.

TABLE I

Possible BACKup PATH CHOICES For $|0,5|^{(0,5)}$

\begin{tabular}{|c||c|c|c|c|}
\hline$j$ & $|s, d|_{1, j}^{(0,5)}$ & $|s, d|_{2, j}^{(0,5)}$ & $D_{S, P}^{j}$ & $P r_{S}^{j}$ \\
\hline 1 & 2 & 0 & 0.02 & 0.81 \\
2 & 1 & 1 & 0.32 & 0.18 \\
3 & 0 & 2 & 1.62 & 0.01 \\
\hline
\end{tabular}

Upon failure of link $\bar{l}=(0,5)$, three working lightpaths $v_{0,5}^{1}$, $v_{0,5}^{2}$, and $v_{1,5}^{1}$ are disrupted. Therefore, the numbers of lightpaths $|s, d|^{\bar{l}}$ in the disrupted lightpath sets are $|0,5|^{(0,5)}=2$ and $|1,5|^{(0,5)}=1$. Moreover, considering the backup paths $r_{0,5}^{1}, r_{0,5}^{2}, r_{1,5}^{1}$, and $r_{1,5}^{2}$ shown in Fig. 1 , the $\lambda_{l}^{(0,5)}$ parameter assumes the following values:

- along $r_{0,5}^{1}: \lambda_{(\underline{\underline{l}}, 1)}^{\bar{l}}=2, \lambda_{(1,4)}^{\bar{l}}=3, \lambda_{(4,5)}^{\bar{l}}=3$;

- along $r_{0,5}^{2}: \lambda_{(0,2)}^{\bar{l}}=2, \lambda_{(2,3)}^{\bar{l}}=3, \lambda_{(3,5)}^{\bar{l}}=3$.

For the failed lightpaths between node pair $(0,5)$, the backup path selection probabilities for the two preplanned backup paths $r_{0,5}^{1}$ and $r_{0,5}^{2}$ are computed at node 0 by utilizing (1) and (2) as

$$
\begin{aligned}
& p_{1}=P_{r_{0,5}^{1}}^{(0,5)}=\frac{9}{10} \\
& p_{2}=P_{r_{0,5}^{2}}^{(0,5)}=\frac{1}{10} .
\end{aligned}
$$

Table I shows, for the three possible disrupted lightpath assignments $j$ to the backup paths $r_{0,5}^{1}$ and $r_{0,5}^{2}$, the values of the following quantities:

- $|s, d|_{i, j}^{\bar{l}}$, with $i \in\{1,2\}$, is the number of failed lightpaths that are assigned to each backup path $r_{0,5}^{i}$;

- $D_{S, P}^{j}$ is the Euclidean distance from the backup path selection probabilities $p_{1}$ and $p_{2}$;

- $\operatorname{Pr}_{S}^{j}$ is the selection probability of the specific assignment if the SPR-PW scheme is utilized. 
The SPR-PW scheme performs independent backup path selections for each disrupted lightpath; therefore, the selection probability $\operatorname{Pr}_{S}^{j}$ of the $j$ th assignment is computed as

$$
\operatorname{Pr}_{S}^{j}\left(|s, d|_{i, j}^{\bar{l}}\right)=\left(\begin{array}{c}
|s, d|^{\bar{l}} \\
|s, d|_{1, j}^{\bar{l}}
\end{array}\right) p_{1}^{|s, d|_{1, j}^{\bar{\tau}}} p_{2}^{|s, d|^{\bar{l}}-|s, d|_{1, j}^{\bar{\tau}}} .
$$

The SPR-PW scheme provides the following average square Euclidean distance from the computed probabilities $p_{1}$ and $p_{2}$ :

$$
\sum_{j=1}^{3} \operatorname{Pr}_{S}^{j} D_{S, P}^{j}=0.09
$$

In comparison, the DPR-PW scheme, in both its exhaustive and iterative implementations, deterministically chooses the solution with the minimum square Euclidean distance from the backup path selection probabilities (i.e., $|s, d|_{1}^{(0,5)}=2$; $\left.|s, d|_{2}^{(0,5)}=0\right)$ and therefore the resulting average square distance is 0.02 .

\section{MSPR-PW and MDPR-PW Schemes}

The MSPR-PW scheme, proposed in [9] as the stochastic integrated multilayer restoration (SIMuR) scheme, is the extension of the SPR-PW scheme to multilayer network architectures. As it can be observed in [6] and [9], the SPR-PW scheme improves its performance (in terms of restoration blocking probability) at a fixed average network throughput ${ }^{4}$ if the number of wavelengths in each link increases. Indeed, intuitively, the higher is the number of available wavelengths per link, the higher is the number of lightpaths that fail simultaneously, and the higher is the number of stochastic choices performed at nodes involved in restoration. Therefore, the fraction of lightpaths assigned to the preplanned backup paths closely approaches their selection probabilities $P_{r_{s, d}^{i}}^{\bar{l}}$, thus potentially minimizing the average restoration blocking probability. The MSPR-PW scheme increases, on purpose, the number of stochastic choices by applying the stochastic choice of the backup path to the lower-order LSPs multiplexed in the disrupted lightpaths.

The MDPR-PW scheme extends the DPR-PW scheme to multilayer network architectures. Similar to the MSPR-PW scheme, the MDPR-PW scheme exploits the possibility of assigning lower-order LSPs, instead of lightpaths, to preplanned backup paths. The increased number of failed connections to be assigned to preplanned backup paths increases the number of possible assignments of failed connections to preplanned paths. Thus, it potentially decreases the minimum achievable distance $D_{S, P}$.

Both MSPR-PW and MDPR-PW can be classified as integrated multilayer restoration schemes because they contemporarily select the path and the granularity (i.e., the network layer) at which lower-order LSPs (e.g., sublightpath connections) multiplexed in failed lightpaths are recovered. Indeed,

\footnotetext{
${ }^{4}$ The network throughput is defined as the ratio between the network capacity utilized by the working lightpaths and the total available network capacity.
}

TABLE II $E\left[\operatorname{Pr}_{b}^{(0,5)}\right]$ VALUES IN FunCtion of $n$

\begin{tabular}{|c||c|c|c|c|c|c|}
\hline$n$ & 1 & 2 & 4 & 8 & 16 & 32 \\
\hline$E\left[P r_{b}^{(0,5)}\right]$ & 0.250 & 0.188 & 0.137 & 0.098 & 0.070 & 0.050 \\
\hline
\end{tabular}

backup paths with high selection probability $P_{r_{s, d}^{i}}^{\bar{l}}$ are more likely to be chosen by disrupted lower-order LSPs. Thus, lowerorder LSPs can be multiplexed in the same backup lightpath and all-optically recovered. On the other hand, LSPs that cannot be multiplexed to fully occupy the lightpath bandwidth are recovered at the sublightpath granularity. However, MSPR-PW and MDPR-PW schemes require additional node capabilities such as node (de)multiplexing (i.e., grooming) capabilities that increase the overall network cost.

1) MSPR-PW Example: In the following example, the potential restoration blocking probability improvement achievable by utilizing the MSPR-PW scheme is shown. Suppose that in the network depicted in Fig. 1 each network link has a capacity of two wavelengths. Four working lightpaths $v_{0,5}^{1}, v_{1,5}^{1}$, $v_{1,4}^{1}$, and $v_{2,3}^{1}$ are established. Therefore, the network utilization parameter values are as follows:

- $c_{l}=2 \forall l$;

- $\mu_{(0,1)}=1, \mu_{(0,5)}=2, \mu_{(1,4)}=1, \mu_{(2,3)}=1$;

- $\mu_{l}=0$ for other links.

Upon failure of link $\bar{l}=(0,5)$, the working lightpaths $v_{0,5}^{1}$ and $v_{1,5}^{1}$ are disrupted. Lightpath $v_{0,5}^{1}$ can be rerouted along either backup path $r_{0,5}^{1}$ or backup path $r_{0,5}^{2}$, while lightpath $v_{1,5}^{1}$ can be rerouted along either $r_{1,5}^{1}$ or $r_{1,5}^{2}$. Network parameter $\lambda_{l}^{(0,5)}$ assumes the following values:

- along $r_{0,5}^{1}: \lambda_{(0,1)}^{\bar{l}}=1, \lambda_{(1,4)}^{\bar{l}}=2, \lambda_{(4,5)}^{\bar{l}}=2$;

- along $r_{0,5}^{2}: \lambda_{(0,2)}^{\bar{l}}=1, \lambda_{(2,3)}^{\bar{l}}=2, \lambda_{(3,5)}^{\bar{l}}=2$;

- along $r_{1,5}^{1}: \lambda_{(1,4)}^{\bar{l}}=2, \lambda_{(4,5)}^{\bar{l}}=2$;

- along $r_{1,5}^{2}: \lambda_{(1,2)}^{\bar{l}}=1, \lambda_{(2,3)}^{\bar{l}}=2, \lambda_{(3,5)}^{\bar{l}}=2$.

From (1) and (2), the backup path selection probabilities are

$$
\begin{aligned}
& p_{1}=P_{r_{0,5}^{1}}^{(0,5)}=P_{r_{1,5}^{1}}^{(0,5)}=\frac{1}{2} \\
& p_{2}=P_{r_{0,5}^{2}}^{(0,5)}=P_{r_{1,5}^{2}}^{(0,5)}=\frac{1}{2} .
\end{aligned}
$$

Given the values of the probabilities $p_{1}$ and $p_{2}$, the expected value of the restoration blocking probability for the MSPR-PW scheme is obtained by averaging among all the possible assignments of the lower-order LSPs multiplexed in the failed lightpaths to the preplanned backup paths

$$
E\left[\operatorname{Pr}_{b}^{(0,5)}\right]=\frac{1}{2 n} \sum_{k=0}^{2 n}\left\{\left(\begin{array}{c}
2 n \\
k
\end{array}\right) p_{1}^{k} p_{2}^{2 n-k}|n-k|\right\}
$$

where $n$ is the number of LSPs in which each of the two disrupted lightpaths is demultiplexed.

If each working lightpath can be demultiplexed into lowerorder LSPs, $E\left[\operatorname{Pr}_{b}^{(0,5)}\right]$ decreases as summarized in Table II. 
At the limit for $n \rightarrow \infty$, the value of $E\left[\operatorname{Pr}_{b}^{(0,5)}\right]$ obtained by utilizing the MSPR-PW scheme approaches 0 . Indeed, for $n \rightarrow \infty$, the disrupted lower-order LSPs are evenly distributed to the two preplanned backup paths. This distribution guarantees the successful restoration of all lower-order LSPs in a full grooming network scenario.

2) Multilayer Restoration Schemes in Next Generation SONET/SDH Over WDM Networks: Next generation SONET/ SDH (NG-S) over wavelength division multiplexing (WDM) networks provide fundamentals for efficiently implementing Data over SONET (DoS) while guaranteeing the coexistence with traditional voice services. NG-S edge nodes, i.e., multiservice provisioning platforms (MSPPs), support generic framing procedure (GFP), virtual concatenation (VCAT), and link capacity adjustment scheme (LCAS) [14]-[17]. The proposed multilayer preplanned restoration schemes (i.e., both MDPRPW and MSPR-PW) can be implemented in NG-S over WDM networks by terminating all optical lines with SONET/SDH Digital Cross Connects (DCS) that demultiplex lightpaths and electrically switch SONET/SDH finer granularity frames that correspond to lower-order LSPs.

For example, consider an NG-S over WDM network, in which each wavelength carries OC-48 (i.e., $2488.32 \mathrm{Mb} / \mathrm{s}$ ) SONET frames. Network nodes are equipped with DCS performing switching functionalities at the DS-3 level (i.e., $44.736 \mathrm{Mb} / \mathrm{s})$. OC-48 capacity is shared between voice and data traffic: one VC-4-7v Virtual Concatenation Groups (VCG) with $1.05 \mathrm{~Gb} / \mathrm{s}$ payload is reserved in each OC-48 for data traffic while the remaining capacity is utilized for voice services. MSPPs are present at each network node. GbE connection requests are generated between MSPPs and mapped into VC-4-7v VCGs. ${ }^{5}$

After failure notification, the MSPPs from which the failed VC-4-7v VCGs originated apply one of the proposed multilayer restoration schemes (e.g., either MDPR-PW or MSPR-PW) at the VC-4 granularity. Thus, each VC-4 belonging to the failed $\mathrm{VC}-4-7 \mathrm{v}$ is separately assigned to a backup path. After the backup path selection, the signaling protocol supporting NG-S (e.g., RSVP-TE signaling) configures the VC-4s along the links spanned by the chosen backup paths [17]. Then, LCAS signaling adds the recovered VC-4s to the same VCG to which the disrupted $\mathrm{VC}-4-7 \mathrm{v}$ belongs.

\section{E. Required Signaling and Recovery Time}

During the lightpath provisioning phase, signaling and routing protocol extensions proposed for the SPR-PW scheme [8], [11] are utilized by all the considered schemes for reserving working lightpath capacity and updating node databases.

During the restoration phase, in all the considered schemes, failure isolation, failure notification, and backup path activation signaling are necessary [18]. Each source node is assumed to discover all the lightpaths disrupted by the failure from a single notification message that carries the ID of the failed link $\bar{l}$.
Two signaling schemes, based on bidirectional mode RSVP-TE signaling [11], are considered for backup path activation: the LSP-oriented and the PATH-oriented signaling schemes. When used in combination with single-layer schemes, LSP-oriented signaling requires one signaling instance per disrupted lightpath to reserve resources along the selected backup path. When used in combination with multilayer schemes, LSPoriented signaling requires one signaling instance per disrupted lower-order LSP to reserve resources along the selected backup path. For each $(s, d)$ pair, the PATH-oriented scheme utilizes one aggregate signaling instance for reserving resources along each backup path selected by at least one disrupted lightpath or lower-order LSP.

While the overhead required by the LSP-oriented signaling does depend on whether a single-layer or a multilayer scheme is implemented, the overhead required by the PATH-oriented signaling does not. Furthermore, the overhead required by both LSP-oriented and PATH-oriented signaling schemes does not depend on the backup path selection strategy (i.e., either stochastic or deterministic).

The recovery time can be expressed as a function of the following quantities:

- $t_{\text {sel }}(j)$ is the computational time needed for selecting the backup path of the $j$ th lightpath or lower-order LSP;

- $t_{\text {sig }}$ is the backup path activation signaling round-trip time;

- $t_{\mathrm{xc}}$ is the time necessary to perform cross connections in nodes belonging to the backup path.

Common values for $t_{\mathrm{sig}}$ and $t_{\mathrm{xc}}$ are in the order of tens of milliseconds and milliseconds respectively [19], [20].

The recovery time experienced by the $j$ th lightpath or lowerorder LSP between the node pair $(s, d)$ depends on both the utilized signaling and restoration schemes. In particular, the recovery time in both single-layer and multilayer stochastic selection schemes utilizing LSP-oriented signaling is

$$
\mathrm{RT}_{j, \mathrm{LSP}, S}^{(s, d)}=t_{\mathrm{sel}}(j)+t_{\mathrm{sig}}+t_{\mathrm{xc}} .
$$

The recovery time in the iterative implementation of both single-layer and multilayer deterministic schemes utilizing LSP-oriented signaling is

$$
\mathrm{RT}_{j, \mathrm{LSP}, D}^{(s, d)}=\sum_{i=1}^{j-1} t_{\mathrm{sel}}(i)+t_{\mathrm{sel}}(j)+t_{\mathrm{sig}}+t_{\mathrm{xc}} .
$$

Equation (12) models the independent backup path selection performed by stochastic schemes for each disrupted lightpath or lower-order LSP. Equation (13) models the fact that, in deterministic schemes, the backup path selection for the $j$ th lightpath or lower-order LSP is performed only upon completion of all the previous $j-1$ backup path selections. Therefore, stochastic schemes that utilize LSP-oriented signaling potentially guarantee fast recovery time.

The recovery time for all the proposed restoration schemes utilizing the PATH-oriented signaling is

$$
\mathrm{RT}_{j, \mathrm{PATH}}^{(s, d)}=\sum_{i=1}^{|s, d|^{\bar{l}}} t_{\mathrm{sel}}(i)+t_{\mathrm{sig}}+t_{\mathrm{xc}} .
$$


TABLE III

Proposed Restoration Schemes AND INVESTIGATED SCENARIOS

\begin{tabular}{c||c|c|c}
\hline & All-Optical & Single-hop & Multihop \\
\hline \hline Stochastic & SPR-PW & MSPR-PW & MSPR-PW \\
\hline Deterministic & DPR-PW & MDPR-PW & MDPR-PW \\
\hline
\end{tabular}

As shown in (14) (where the sum is extended to all disrupted lightpaths or lower-order LSPs), the signaling instance is activated once all the disrupted lightpaths or lower-order LSPs have been assigned to a backup path. Therefore, the recovery time is potentially higher than that of schemes utilizing LSP-oriented signaling.

\section{Performance Evaluation}

\section{A. Scenarios}

A multilayer optical network with a GMPLS control plane is considered (e.g., NG-S over WDM). During the provisioning phase, connections between each $(s, d)$ pair are generated and routed at the lightpath granularity. Thus, lightpath capacity (e.g., OC-48) is fully utilized. Upon failure occurrence, after failure localization and failure notification, source network nodes run one of the proposed preplanned restoration schemes and then trigger the restoration signaling to recover disrupted connections.

The performance of the investigated restoration schemes is evaluated in three different grooming scenarios summarized in Table III. In the all-optical scenario, network nodes do not have grooming capabilities. Thus, lightpaths are recovered as a whole at the optical layer. In single-hop grooming and multihop grooming scenarios, disrupted lightpaths can be recovered by exploiting network node grooming capabilities. In particular, in the single-hop grooming scenario, just the network edge nodes are equipped with grooming capabilities. Each disrupted lower-order LSP is recovered by establishing or by reutilizing single-hop lightpaths between the same node pairs connected by the disrupted lightpaths. Thus, only disrupted lower-order LSPs belonging to the same node pair $(s, d)$ can be multiplexed in lightpaths activated along the selected backup paths. In the multihop grooming scenario, grooming capabilities of intermediate nodes along a backup path are exploited. Disrupted lower-order LSPs are recovered by establishing or by reutilizing one or more single-hop lightpaths along the selected backup paths. Lower-order LSPs belonging to different $(s, d)$ pairs can be multiplexed into the same lightpath by utilizing electronic grooming capabilities of any node along the selected backup paths. Thus, in the multihop grooming scenario, a more efficient network capacity utilization is achievable. However, equipping all the nodes with grooming capabilities leads to a significant increase in the overall network cost.

\section{B. Methodology}

The performance evaluation of the proposed schemes is based on three main parameters: the restoration blocking probability, the restoration signaling overhead, and the idle restoration capacity.
Given the failure of a bidirectional link $\bar{l} \in \mathcal{L}$, the restoration blocking probability $\operatorname{Pr}_{b}^{\bar{l}}$ conditional to the failure of link $\bar{l}$ is defined as the ratio between the number $\operatorname{LSP}_{u}^{\bar{l}}$ of unrecovered lower-order LSPs and the total number $\operatorname{LSP}_{f}^{\bar{l}}$ of disrupted lower-order LSPs

$$
\operatorname{Pr}_{b}^{\bar{l}}=\frac{\operatorname{LSP}_{u}^{\bar{l}}}{\operatorname{LSP}_{f}^{\bar{l}}}
$$

The restoration blocking probability $\overline{\operatorname{Pr}}_{b}$ is defined as the average of the conditional restoration blocking probability over all the possible single link failures

$$
\overline{\operatorname{Pr}}_{b}=\sum_{\bar{l}=0}^{|\mathcal{L}|-1} \operatorname{Pr}_{f}^{\bar{l}} \operatorname{Pr}_{b}^{\bar{l}}
$$

where $\operatorname{Pr}_{f}^{\bar{l}}$ is the failure probability of link $\bar{l}$.

The restoration signaling overhead is represented by the number of hops spanned by the PATH and RESV messages utilized for lower-order LSPs restoration averaged over all the possible single-link failures. The LSP-oriented signaling overhead $O_{\text {LSP }}$ and the PATH-oriented signaling overhead $O_{\mathrm{PATH}}$ are defined, respectively, as

$$
\begin{aligned}
O_{\mathrm{LSP}} & =\sum_{\bar{l}=0}^{|\mathcal{L}|-1} \operatorname{Pr}_{f}^{\bar{l}} \sum_{s, d} \sum_{i=1}^{k} 2 h_{r_{s, d}^{i}} \operatorname{LSP}_{f, r_{s, d}^{i}}^{\bar{l}} \\
O_{\mathrm{PATH}} & =\sum_{\bar{l}=0}^{|\mathcal{L}|-1} \operatorname{Pr}_{f}^{\bar{l}} \sum_{s, d} \sum_{i=1}^{k} 2 h_{r_{s, d}^{i}} N_{i} .
\end{aligned}
$$

In (17), $\operatorname{LSP}_{f, r_{s, d}^{i}}^{\bar{l}}$ is the number of disrupted lower-order LSPs between the node pair $(s, d)$ that are recovered along the path $r_{s, d}^{i}$. In (18), $N_{i}$ is a binary variable assuming the value of 1 if one or more lower-order LSPs are assigned to backup path $r_{s, d}^{i}$ and 0 otherwise. In (17) and (18), $h_{r_{s, d}^{i}}$ represents the number of links spanned by the $i$ th backup path $r_{s, d}^{i}$. Finally, the factor 2 takes into account the reservation message round trip. Equation (18) also represents the average signaling overhead when a single-layer restoration scheme is utilized.

The third evaluation parameter is the idle restoration capacity $I_{\mathrm{RC}}$ that measures the unutilized lightpath bandwidth after the restoration attempt in multihop grooming scenarios. The value of $I_{\mathrm{RC}}$ is computed after the restoration attempt by averaging the link idle restoration capacity over all the network bidirectional links

$$
I_{\mathrm{RC}}=\frac{1}{|\mathcal{L}|} \sum_{p \in \mathcal{P}_{r}} u_{p} h_{p}
$$

where $\mathcal{P}_{r}$ is the set of lightpaths activated during the restoration phase while $u_{p}$ and $h_{p}$ represent, respectively, the unutilized lightpath $p$ bandwidth and the number of links spanned by the lightpath. 


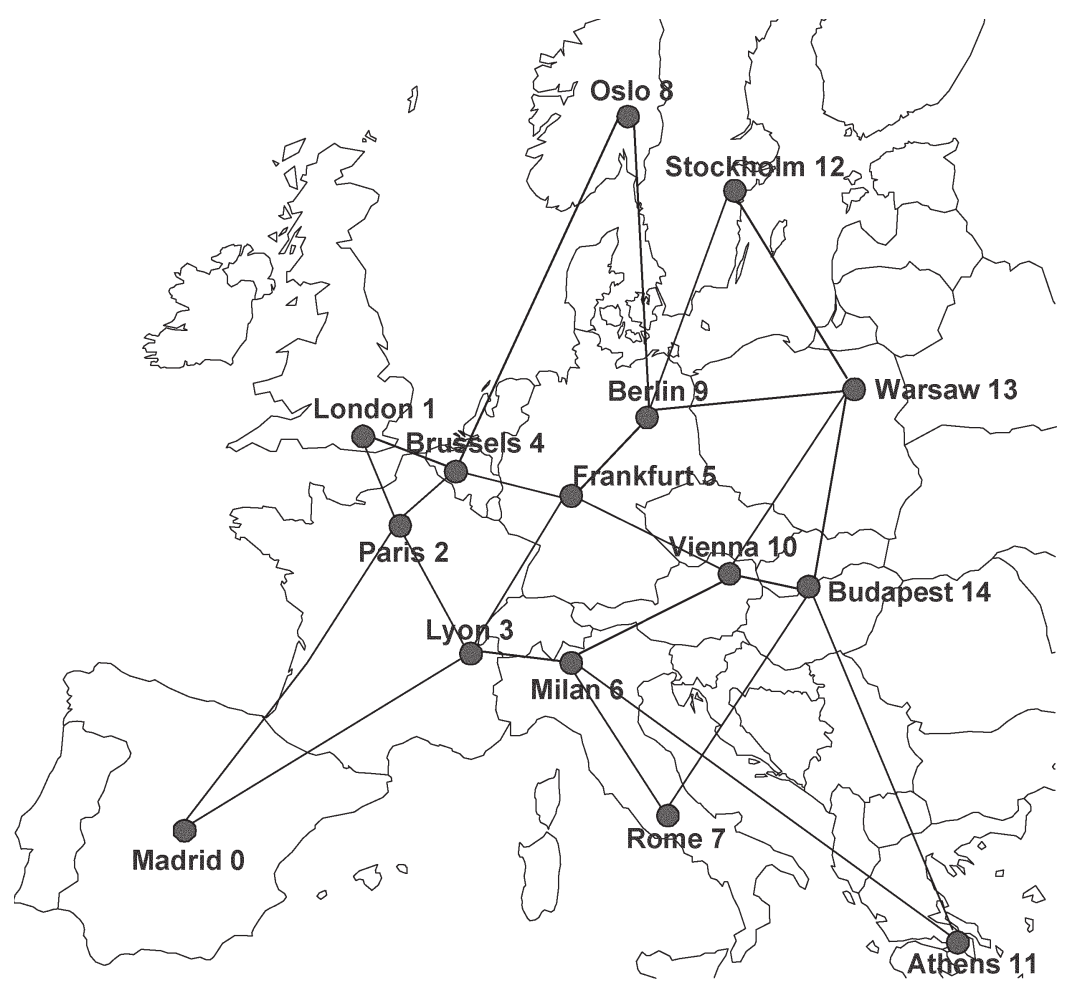

Fig. 2. Pan-European network topology.

\section{NumericAl Results}

The proposed restoration schemes are evaluated in the PanEuropean network topology, depicted in Fig. 2, by using a custom-built simulator. The Pan-European network consists of 15 nodes and 24 bidirectional links. Each network node is assumed to have full wavelength conversion capabilities and the total capacity of each link $c_{l}$ is set to 32 wavelengths.

The preplanned restoration schemes outlined in Section II are also compared against the optimal solution of the linear programming (LP) formulation of the PRR problem adaptation to multilayer networks with given link capacity and grooming capable nodes (i.e., multihop grooming scenario). The considered problem optimal solution is the one maximizing the number of recovered lower-order LSPs under the assumption that failed lightpaths can be demultiplexed into an infinite number of lower-order LSPs. Thus, the average restoration blocking probability obtained by the optimal solution represents the lower bound for all the investigated restoration schemes.

Each experiment consists of generating a set of lightpath patterns, uniformly distributed among all the possible node pairs $(s, d)$, until a specific average network throughput is reached. The network throughput is defined as the ratio between the network capacity utilized by the working lightpaths and the total available network capacity.

For each experiment, all the possible bidirectional link failures are generated and the investigated recovery schemes are applied. The link failure probability $\operatorname{Pr}_{f}^{\bar{l}}$ is assumed to be uniformly distributed among all the bidirectional links (i.e., $\operatorname{Pr}_{f}^{\bar{l}}=$ $1 /|\mathcal{L}|)$. The values of the performance evaluation parameters are then computed and averaged among all the experiments repeated by generating different lightpath patterns achieving

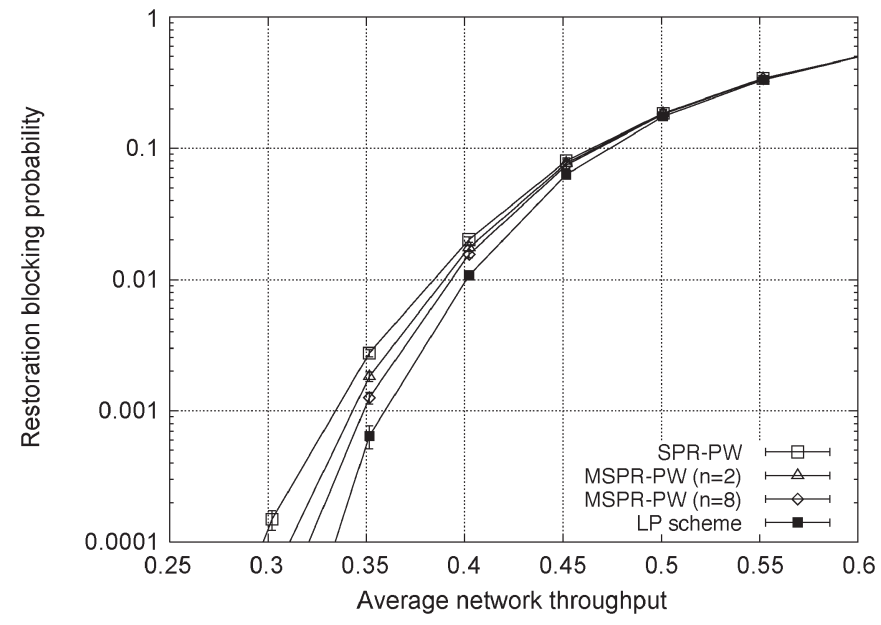

Fig. 3. Comparison between SPR-PW and MSPR-PW (multilayer restoration) in multihop grooming scenario utilizing $k=2$ preplanned backup paths.

the same network throughput. For each set of experiments, the confidence interval of the performance evaluation parameters is computed with the $95 \%$ confidence level.

Figs. 3 and 4 compare the performance of the investigated schemes in terms of restoration blocking probability $\overline{\operatorname{Pr}}_{b}$ as a function of the average network throughput when $k=2$ preplanned backup paths are available in the multihop grooming scenario. For the DPR-PW scheme, only the results regarding the I-DPR-PW implementation are shown because of the proved equivalence between the E-DPR-PW and the I-DPR-PW in this case (for the details, see Appendix). Fig. 3 shows that, by utilizing upper-layer functionalities of the MSPR-PW, it is possible to decrease the restoration blocking probability at 


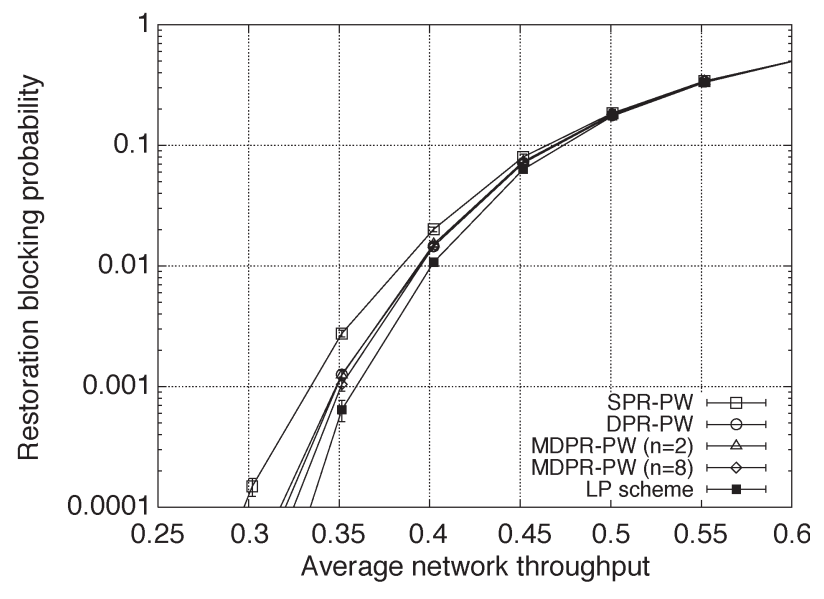

Fig. 4. Comparison between SPR-PW, DPR-PW (single layer), and MDPR$\mathrm{PW}$ (multilayer) in multihop grooming scenario utilizing $k=2$ preplanned backup paths.

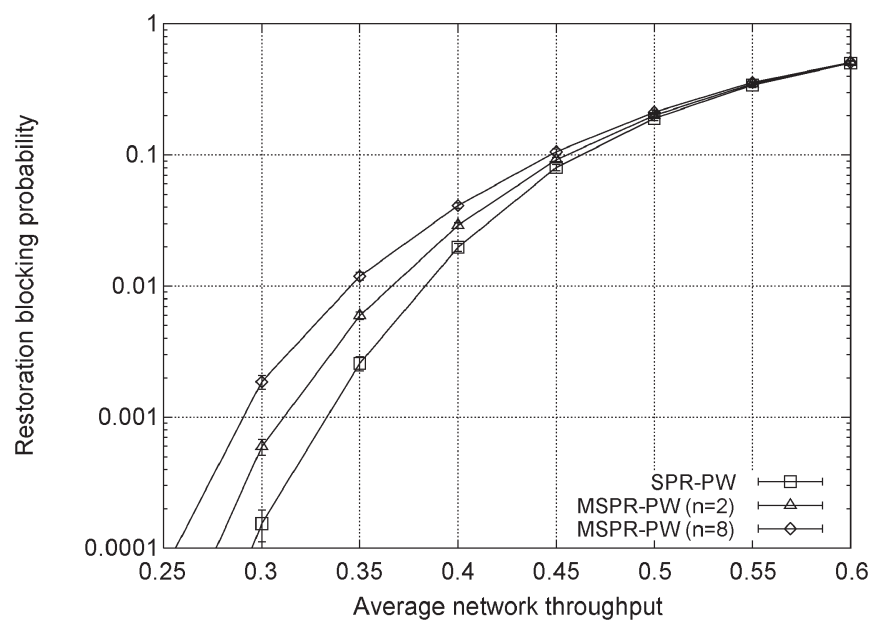

Fig. 5. Comparison between SPR-PW and MSPR-PW (multilayer) in singlehop grooming scenario utilizing $k=2$ preplanned backup paths.

any network throughput and closely approximate the optimal solution given by the LP scheme. However, due to the limited number of preplanned backup paths, the restoration blocking probability improvement, expected from (11), is slight. Fig. 4 shows that the DPR-PW scheme is able to achieve a restoration blocking probability very close to that obtained by the MSPR-PW scheme without requiring node grooming capabilities. Moreover, as shown in Fig. 4, the utilization of the MDPR-PW does not substantially improve the restoration blocking probability even in a multihop grooming scenario.

Figs. 5 and 6 compare the performance of the investigated schemes in terms of restoration blocking probability as a function of the average network throughput in the single-hop grooming scenario when $k=2$ preplanned restoration paths are available. Figs. 5 and 6 show that, in the single-hop grooming scenario, the utilization of the considered multilayer preplanned restoration schemes (i.e., MSPR-PW and MDPR-PW) does not improve the restoration blocking probability obtained by the SPR-PW. In addition, Fig. 6 shows that even in the singlehop grooming scenario, DPR-PW obtains the best performance among both single-layer and multilayer deterministic and stochastic schemes.

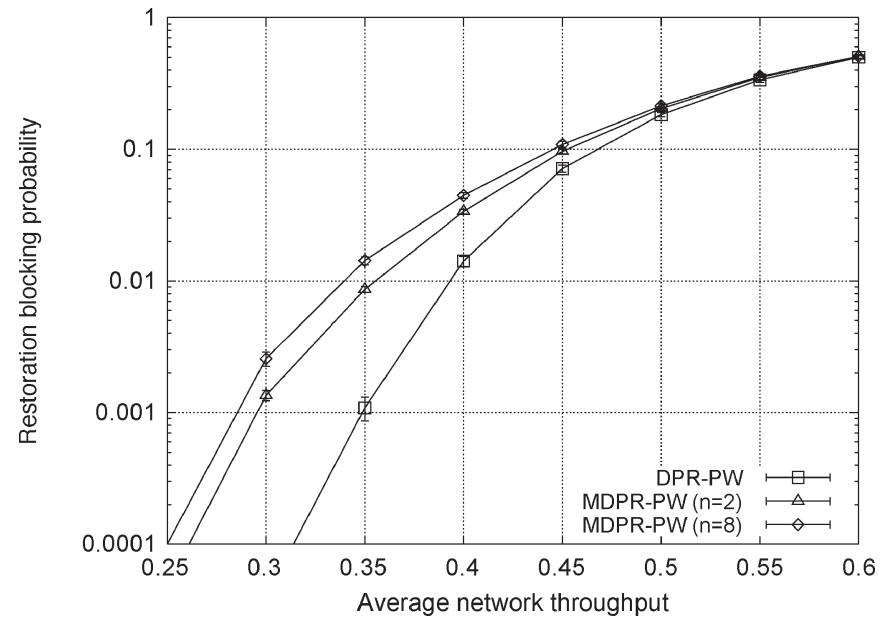

Fig. 6. Comparison between DPR-PW (single layer) and MDPR-PW (multilayer) in single-hop grooming scenario utilizing $k=2$ preplanned backup paths.

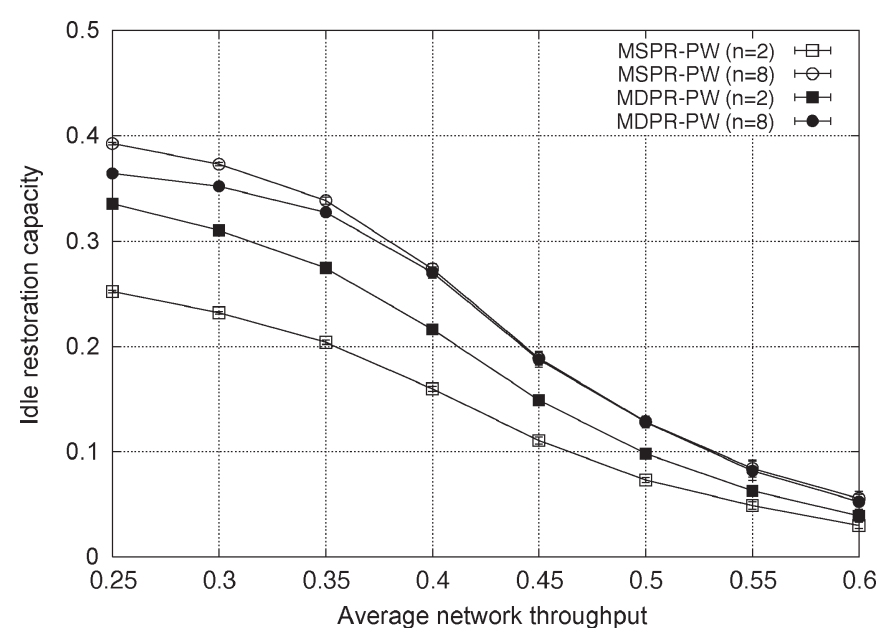

Fig. 7. Idle restoration capacity with $k=2$ preplanned backup paths for each working lightpath and single-hop grooming.

In addition, Figs. 5 and 6 show that, for all the considered multilayer schemes, as the number $n$ of lower-order LSPs multiplexed in a single lightpath increases, the restoration blocking probability increases too. As shown in Fig. 7, this is due to the lower efficiency in the utilization of end-to-end lightpaths by fine-granularity lower-order LSPs (i.e., high idle restoration capacity). Indeed, in the single-hop grooming scenario, just lower-order LSPs between the same $(s, d)$ pair may share lightpaths activated along the chosen backup path.

Figs. $8-11$ show the results obtained by the investigated multilayer and single-layer preplanned restoration schemes when three backup paths are preplanned for each working lightpath $(k=3)$. Even in this case, only the I-DPR-PW implementation of the DPR-PW scheme is considered. If three paths are utilized, the performance improvement with respect to the SPR-PW scheme in the multihop grooming scenario increases for all the investigated schemes. In addition, the relative behavior of the investigated schemes obtained with $k=2$ is confirmed.

Fig. 12 shows that PATH-oriented signaling is more scalable than LSP-oriented signaling in function of the number $n$ of 


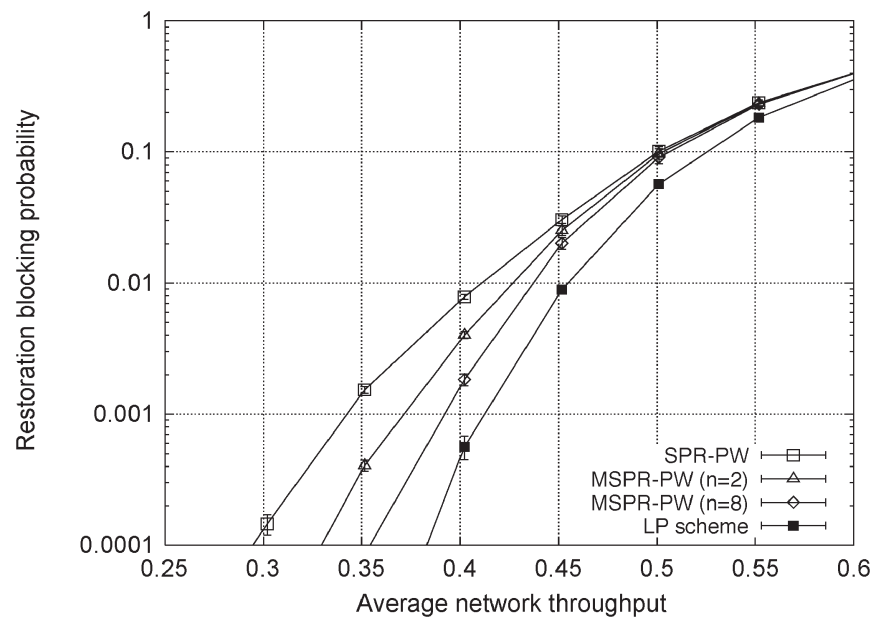

Fig. 8. Comparison between SPR-PW and MSPR-PW (multilayer) in multihop grooming scenario utilizing $k=3$ preplanned backup paths.

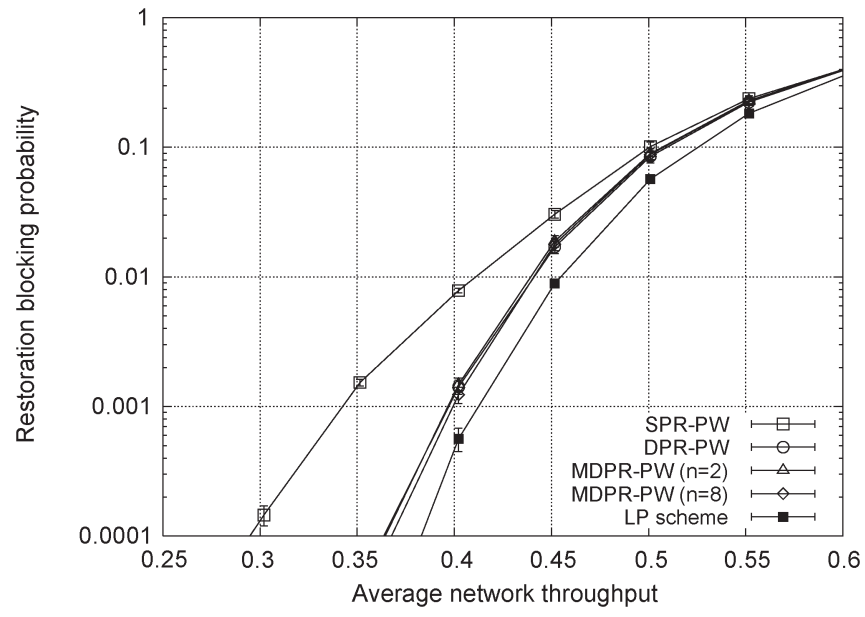

Fig. 9. Comparison between SPR-PW, DPR-PW (single layer), and MDPR$\mathrm{PW}$ (multilayer) in multihop grooming scenario utilizing $k=3$ preplanned backup paths.

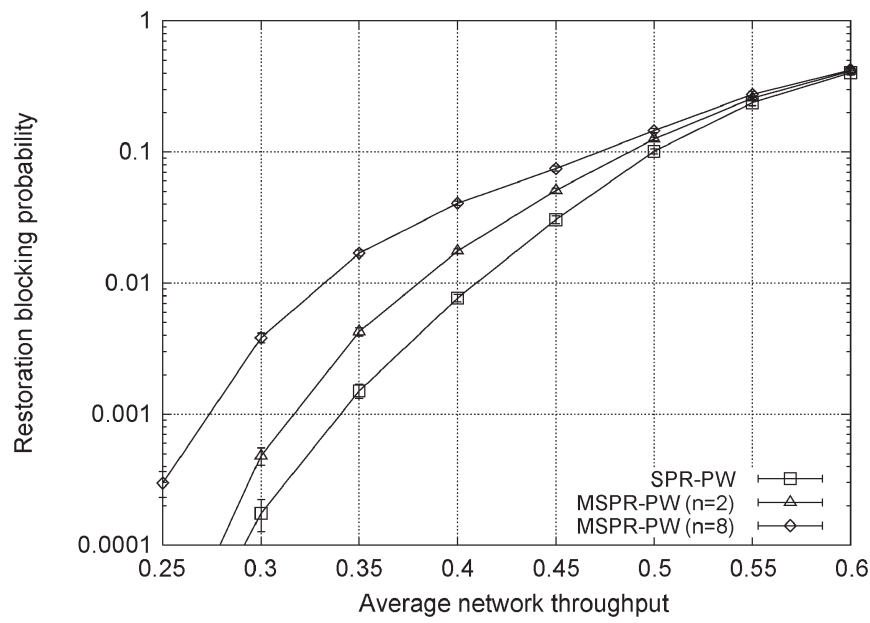

Fig. 10. Comparison between SPR-PW and MSPR-PW (multilayer) in single-hop grooming scenario utilizing $k=3$ preplanned backup paths.

LSPs multiplexed into the failed lightpaths. As depicted in Fig. 12, the signaling overhead required by the LSP-oriented signaling increases linearly as a function of the number of

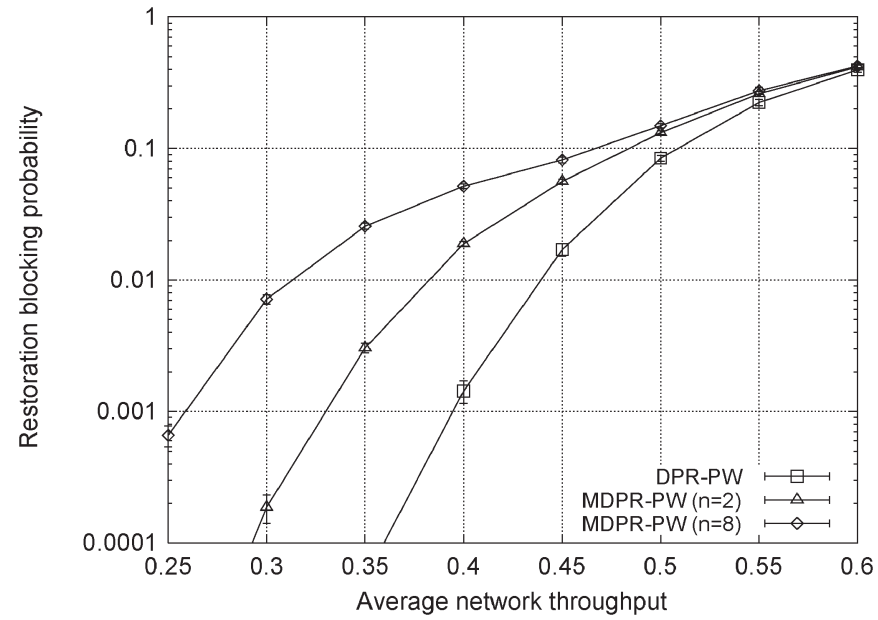

Fig. 11. Comparison between DPR-PW and MDPR-PW (multilayer) in single-hop grooming scenario.

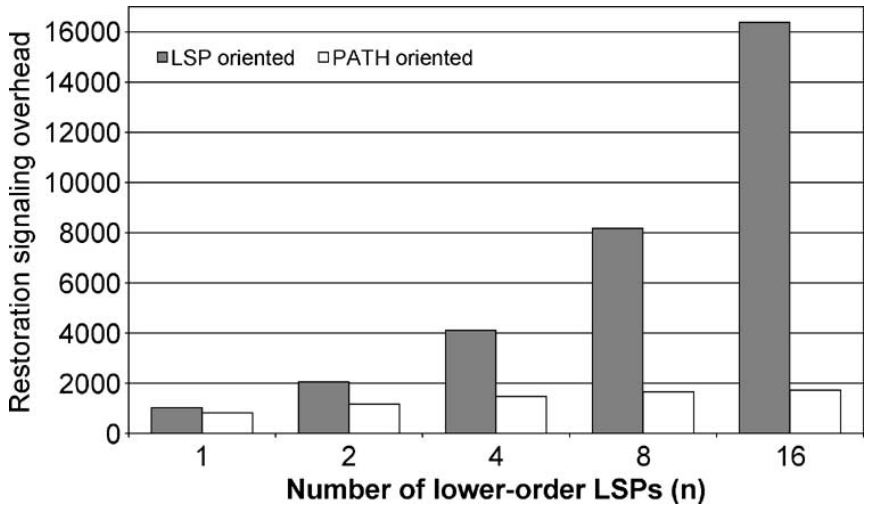

Fig. 12. Signaling overhead at average network throughput 0.5 utilizing $k=3$ preplanned backup paths.

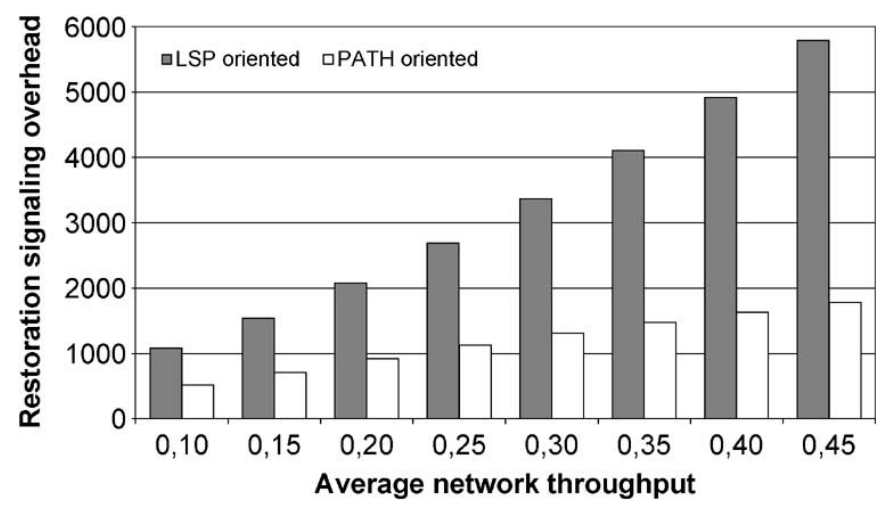

Fig. 13. Signaling overhead with $n=4$ utilizing $k=3$ preplanned backup paths.

LSPs multiplexed into failed lightpaths while the average PATH-oriented signaling overhead increases only slightly. This behavior could be expected by comparing (17) and (18). Furthermore, Fig. 13 shows a slight increase of the signaling overhead for the PATH-oriented signaling scheme as a function of increasing average network throughput. This increase is due to the higher number of failed lightpaths. However, the increase is small because of the high number of backup paths that are 
not selected, and thus not activated, due to unavailable spare resources along their links.

\section{SUMMARY}

In this paper, a family of deterministic preplanned restoration schemes has been proposed. The novelty of the proposed schemes is a deterministic method for selecting the preplanned backup path utilized to recover disrupted lightpaths. As in already proposed stochastic preplanned restoration schemes, the information utilized by the methods consists of the network state at the failure occurrence and on the forecast network link occupation after lightpath recovery. Single-layer deterministic preplanned restoration with proportional weighted path choice (DPR-PW) and multilayer DPR-PW (MDPR-PW) schemes have been compared against single-layer [stochastic preplanned restoration with proportional weighted path choice (SPR-PW)] and multilayer [multilayer SPR-PW (MSPR-PW)] implementations of the stochastic preplanned restoration with proportional weighted path choice (SPR-PW) scheme.

The DPR-PW scheme has shown the ability of closely approximating the optimal assignment of failed lightpaths to the preplanned backup paths if lightpath capacity is fully utilized. Moreover, in all the analyzed network scenarios characterized by different network node grooming capabilities, the DPR-PW scheme has guaranteed restoration blocking probabilities better than or similar to those of both single-layer and multilayer stochastic and multilayer deterministic preplanned restoration schemes. The DPR-PW scheme does not require additional signaling with respect to the SPR-PW scheme during lightpath recovery and does not require the interlayer coordination needed in multilayer schemes. On the other hand, the DPR-PW scheme recovery time is slightly higher than that of stochastic schemes because of the required intranode coordination during backup path selection.

Thus, the proposed deterministic preplanned restoration schemes represent an appealing solution for lightpath recovery. In particular, if lightpaths are fully utilized, it has been shown that it is highly efficient to utilize the single-layer DPR-PW scheme because it guarantees optimal performance without impacting the overall network cost.

\section{APPENDIX}

\section{PRoOF OF E-DPR-PW AND I-DPR-PW EQUIVALENCE}

The objective of this Appendix is to prove the equivalence between the two implementations of the DPR-PW scheme (i.e., E-DPR-PW and I-DPR-PW) when $k=2$ preplanned backup paths $r_{1}$ and $r_{2}$ are available to disrupted connections belonging to a specific $(s, d)$ node pair. The number of connections between the $(s, d)$ pair disrupted by the failure is assumed to be $|s, d|=N$. In this scenario, the possible distribution of the $N$ failed connections to the two preplanned backup paths is $\left(\begin{array}{c}|s, d|+k-1 \\ k-1\end{array}\right)=N+1$. The following quantities are defined:

- $\mathcal{Z}^{N}=\left\{\vec{z}_{0}^{N}, \vec{z}_{1}^{N}, \ldots, \vec{z}_{m}^{N}, \ldots, \vec{z}_{N}^{N}\right\}$ is the set of $N+1$ possible distributions of failed connections to the preplanned backup paths;

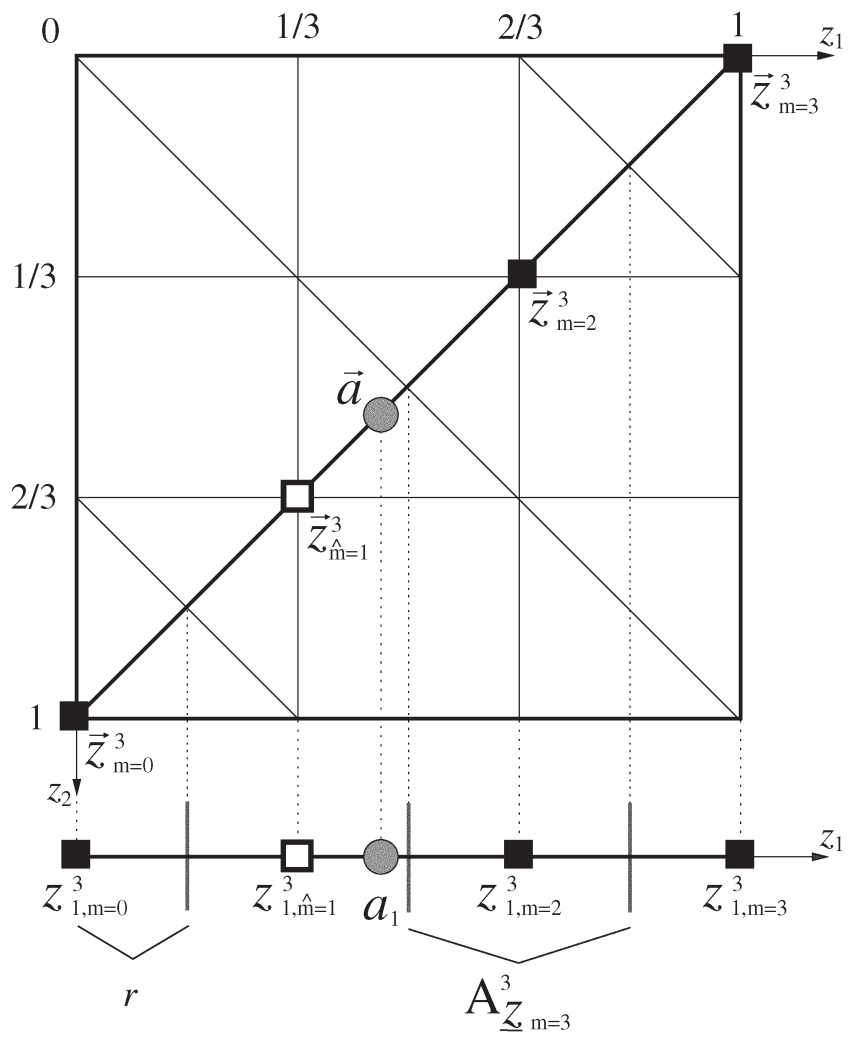

Fig. 14. Considered fraction of the real plane and vector projections on the $z_{1}$ axis for $N=3$.

- $\vec{z}_{m}^{N}=\left(z_{1, m}^{N}, z_{2, m}^{N}\right)=(1 /|s, d|)(m, N-m)=(1 /|s, d|)$. $\left(|s, d|_{1},|s, d|_{2}\right)$ is the vector representing one possible distribution of failed connections to the preplanned backup paths normalized to the number $|s, d|$ of failed connections between the $(s, d)$ pair;

- $\vec{a}=\left(a_{1}, a_{2}\right)=\left(P_{r_{1}}, P_{r_{2}}\right) \in \mathbb{R}^{2}$ is the objective point consisting of the computed path selection probability pair;

- $\vec{z}_{\widehat{m}}^{N} \in \mathcal{Z}^{N}$ is the vector representing the failed connection distribution selected by either the E-DPR-PW or the I-DPR-PW.

Given the former definitions, the constraint formulated in (3) becomes

$$
\sum_{i=1}^{2} z_{i, m}^{N}=1, \quad z_{i, m} \geq 0 \quad \forall m, \forall N .
$$

The minimization problem formulated in (4) is equivalent, in this scenario, to find the vector $\vec{z}_{\widehat{m}}^{N} \in \mathcal{Z}^{N}$ whose distance from the objective point $\vec{a}$ is minimal

$$
\vec{z}_{\widehat{m}}^{N}=\min _{m}\left\|\vec{z}_{m}^{N}-\vec{a}\right\|^{2} .
$$

The solution space of the problem in (21) is represented by the set $\mathcal{Z}^{N}$ and is contained in the fraction of the real plane constrained by the inequalities $0 \leq z_{1} \leq 1$ and $0 \leq z_{2} \leq 1$ as depicted in Fig. 14 for $N=3$. The generic solution $\vec{z}_{m}^{N}$ belongs to the segment going from the point with coordinates $\left(z_{1}=1\right.$, $\left.z_{2}=0\right)$ to the point with coordinates $\left(z_{1}=0, z_{2}=1\right)$ of the solution space. Because all the failed connections must be assigned to a preplanned backup path, a solution may also be 


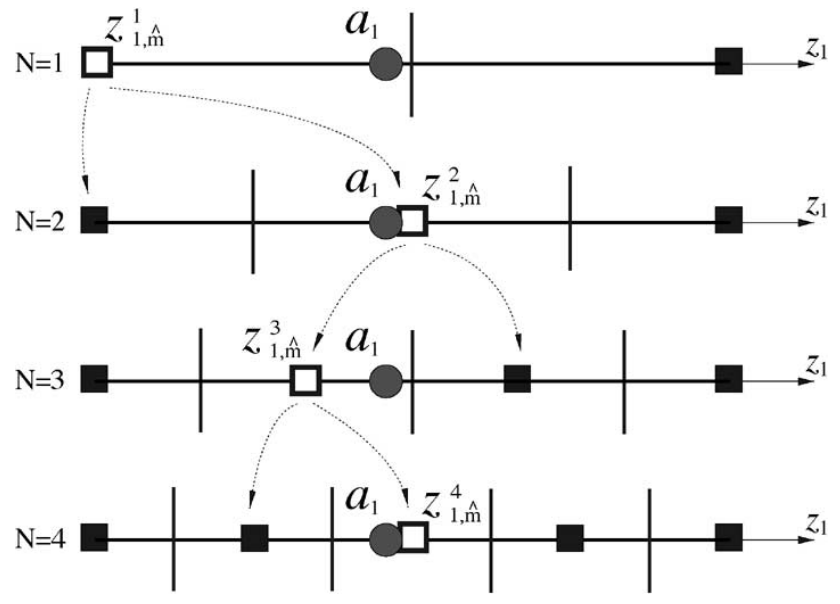

Fig. 15. Vector projections on the $z_{1}$ axis for $N=1,2,3,4$. The empty square represents the selected solution for $N$ while the dotted arrows point the selectable solution at step $N+1$ by I-DPR-PW implementation.

represented by projecting the vector $\vec{z}_{m}^{N}$ on one of the axes (e.g., $z_{1}$ ), i.e., by the number of connections assigned to one preplanned backup path (e.g., $r_{1}$ ).

In Fig. 14, the circle represents $\vec{a}$ while squares represent elements of the set $\mathcal{Z}^{3}$. Due to the constraint in (20) and considering that $|s, d|_{i} \in \mathbf{N}$, the set $\mathcal{Z}^{3}$ contains $N+1=4$ elements. In particular, $\mathcal{Z}^{3}=\{(0,1),(1 / 3,2 / 3),(2 / 3,1 / 3),(1,0)\}$.

$A_{\vec{z}_{m}}^{N}$ represents the decision segment (delimited by the vertical bars in Fig. 14) proper of the specific solution $\vec{z}_{m}^{N}$. The decision segment contains the set of points for which $\vec{z}_{m}^{N}$ is the closest feasible solution. Therefore, if the projection of $\vec{a}$ is contained in the decision segment $A_{\vec{z}_{m}}^{N}, \vec{z}_{m}^{N}$ is the closest solution to the objective point $\vec{a}$.

In the scenario depicted in Fig. 14, the E-DPR-PW scheme selects $\vec{z}_{\hat{m}=1}^{3}=(1 / 3,2 / 3)$ (i.e., the empty square in Fig. 14) as the solution minimizing the square distance from $\vec{a}$. In fact, by projecting all the feasible solutions and $\vec{a}$ on the $z_{1}$ axis, the projection of $\vec{a}$ is in the decision segment relative to the solution $\vec{z}_{m=1}^{3}$.

E-DPR-PW and I-DPR-PW implementations result in equivalence if they choose the same solution $\vec{z}_{\widehat{m}}^{N}$ for any value of $|s, d|=N$. The equivalence between E-DPR-PW and I-DPR-PW can be proved by induction. Thus, the following statements must be proved.

1) If $|s, d|=1$, the two scheme implementations choose the same solution $\vec{z}_{\widehat{m}}^{1}$.

2) If I-DPR-PW chose the same solution $\vec{z}_{\widehat{m}}^{N}$ chosen by E-DPR-PW for $|s, d|=N$, it chooses the same solution also for $|s, d|=N+1$.

When $|s, d|=1, \mathcal{Z}^{1}$ holds two possible solutions $\mathcal{Z}^{1}=$ $\{(0,1),(1,0)\}$. Both implementations, driven respectively by (4) and (5), choose the solution $\vec{z}_{\widehat{m}}^{1}$ that minimizes the square distance from $\vec{a}$.

If $|s, d|=N, \mathcal{Z}^{N}$ contains $N+1$ possible solutions and therefore the projection axis $z_{1}$ is divided in $N+1$ decision segments as depicted Fig. 15.

For $|s, d|=N$, the decision segment radius $r=1 / 2 N$ is defined as the distance between the projection of the generic solution $\vec{z}_{m}^{N}$ and its decision segment bounds. Thus, the coordinate along the projection axis $z_{1}$ of the $m$ th element of $\mathcal{Z}^{N}$, with $m=0,1, \ldots, N$ is

$$
z_{1, m}^{N}=2 r m .
$$

The decision segments $A_{\vec{z}_{m}}^{N}$ have the following bounds:

- $A_{z_{m}}^{N}=[0, r)$ if $m=0$

- $A_{z_{m}}^{N}=[2 r m-r, 2 r m+r)$ if $m \in\{1,2, \ldots, N-1\}$

- $A_{\vec{z}_{m}}^{N}=[1-r, 1]$ if $m=N$.

By assumption, I-DPR-PW and E-DPR-PW choose for $|s, d|=N$ the same solution $\vec{z}_{\widehat{m}}^{N}$. If $|s, d|=N+1$, E-DPR-PW can choose any of the possible $N+2$ solutions. Instead, as shown by the dotted arrows in Fig. 15, I-DPR-PW can choose only among the solutions minimizing the square distance from $\vec{a}$ and contained in the subset $\mathcal{S}^{N+1} \subset \mathcal{Z}^{N+1}$ as

$$
\mathcal{S}^{N+1}=\left\{\vec{z}_{\tilde{m}}^{N+1}, \vec{z}_{\vec{m}+1}^{N+1}\right\}
$$

where $\widetilde{m}$ represents the index of the solution selected for $|s, d|=N$. Therefore, it is sufficient to prove that the solution minimizing the square distance from $\vec{a}$ for $|s, d|=N+1$ belongs to the subset $\mathcal{S}^{N+1}$ (i.e., $\vec{z}_{\widehat{m}}^{N+1} \in \mathcal{S}^{N+1}$ ). This is equivalent to prove the following proposition:

Proposition 1: If $\vec{a} \in A_{\vec{z} \widehat{m}}^{N} \Rightarrow \vec{a} \in\left\{A_{\vec{z} \tilde{m}^{\prime}}^{N+1} \cup A_{\vec{z} \widetilde{m}+1}^{N+1}\right\}$.

Proof: The former proposition is proved by calculating the bounds of decision segments $A_{\vec{z}_{\tilde{m}}}^{N}, A_{\vec{z}_{\tilde{m}}}^{N+1}$, and $A_{\vec{z}_{\tilde{m}+1}^{N+1}}^{N+}$. Considering the decision segment bounds, it results in

$$
A_{\vec{z}_{\tilde{m}}}^{N} \subset\left\{\underset{\vec{z}_{m}}{N+1} \cup A_{\vec{z}_{m}}^{N+1}\right\} \quad \forall m
$$

and therefore Proposition 1 results in true $\forall m$ and $\forall N$.

\section{ACKNOWLEDGMENT}

The authors would like to thank Prof. L. Kazovsky for his helpful suggestions during paper revision.

\section{REFERENCES}

[1] C. Ou, K. Zhu, H. Zang, L. H. Sahasrabuddhe, and B. Mukherjee, "Traffic grooming for survivable WDM networks-Shared protection," IEEE J. Sel. Areas Commun., vol. 21, no. 9, pp. 1367-1383, Nov. 2003.

[2] S. Darisala, A. Fumagalli, P. Kothandaraman, M. Tacca, and L. Valcarenghi, "Multi-failure survivability in optical networks," in Proc. Photonic Switching (PS), Paris, France, 2003, pp. 168-170.

[3] J. Wang, L. H. Sahasrabuddhe, and B. Mukherjee, "Path vs. subpath vs. link restoration for fault management in IP-over-WDM networks: Performance comparisons using GMPLS control signaling," IEEE Commun. Mag., vol. 40, no. 11, pp. 80-87, Nov. 2002.

[4] M. Clouqueur and W. Grover, "Availability analysis of span-restorable mesh networks," IEEE J. Sel. Areas Commun., vol. 20, no. 4, pp. 810 821, May 2002.

[5] H. Lee, J. Chung, and S.-J. Chung, "A state-dependent preplanned ATM VP restoration scheme," in Proc. Global Telecommunications (GLOBECOM), Sydney, N.S.W., Australia, Nov. 1998, pp. 1766-1771.

[6] A. Fumagalli and L. Valcarenghi, "The preplanned weighted restoration scheme," in Proc. IEEE Workshop High Performance Switching and Routing (HPSR), Dallas, TX, 2001, pp. 36-41.

[7] R. Iraschko and W. Grover, "A highly efficient path-restoration protocol for management of optical network transport integrity," IEEE J. Sel. Areas Commun., vol. 18, no. 5, pp. 779-794, May 2000. 
[8] L. Valcarenghi and A. Fumagalli, "Implementing stochastic pre-planned restoration with proportional weighted path choice in IP/GMPLS networks," Photonic Netw. Commun., vol. 4, no. 3/4, pp. 285-295, Jul./Dec. 2002.

[9] P. Castoldi, F. Cugini, A. Giorgetti, and L. Valcarenghi, "An integrated scheme for multilayer network restoration," in Proc. Photonic Switching (PS), Paris, France, 2003, pp. 171-173.

[10] A. Banerjee, J. Drake, J. Lang, L. Turner, B. K. Kompella, and Y. Rekhter, "Generalized multiprotocol label switching: An overview of routing and management enhancements," IEEE Commun. Mag., vol. 39, no. 1, pp. 144-150, Jan. 2001.

[11] A. Banerjee, J. Drake, J. Lang, B. Turner, D. Awduche, L. Berger, K. Kompella, and Y. Rekhter, "Generalized multiprotocol label switching: An overview of signaling enhancements and recovery techniques," IEEE Commun. Mag., vol. 39, no. 7, pp. 144-151, Jul. 2001.

[12] H. Zhu, H. Zang, K. Zhu, and B. Mukherjee, "A novel generic graph model for traffic grooming in heterogeneous WDM mesh networks," IEEE/ACM Trans. Netw., vol. 11, no. 2, pp. 285-299, Apr. 2003.

[13] S. Ramamurthy, L. Sahasrabuddhe, and B. Mukherjee, "Survivable WDM mesh network," J. Lightw. Technol., vol. 21, no. 4, pp. 870-883, Apr. 2003.

[14] S. Kartalopoulos, Next Generation SONET/SDH. Piscataway, NJ: IEEE Press, 2004.

[15] E. Hernandez-Valencia, M. Scholten, and Z. Zhu, "The generic framing procedure (GFP): An overview," IEEE Commun. Mag., vol. 40, no. 5, pp. 63-71, May 2002.

[16] P. Bonenfant and A. Rodriguez-Moral, "Generic framing procedure (GFP): The catalyst for efficient data over transport," IEEE Commun. Mag., vol. 40, no. 5, pp. 72-79, May 2002.

[17] D. Cavendish, K. Murakami, S.-H. Yun, O. Matsuda, M. Nishihara, "New transport services for next-generation SONET/SDH systems," IEEE Commun. Mag., vol. 40, no. 5, pp. 80-87, May 2002.

[18] J. Yates and G. Li, "Challenges in intelligent transport network restoration," in Proc. Optical Fiber Communication (OFC), Atlanta, GA, Mar. 2003, pp. 773-774.

[19] M. Goyal, J. Yates, G. Li, and W. Feng, "Benefits of restoration signaling aggregation," in Proc. Optical Fiber Communication (OFC), Atlanta, GA, 2003, pp. 203-204.

[20] F. Cugini, L. Valcarenghi, P. Castoldi, and P. Raponi, "A costeffective implementation of fast GMPLS shared protection for IP over 10 gigabit Ethernet networks," in IEEE Workshop High Performance Switching and Routing (HPSR), Hong Kong, 2005, pp. 100-103.

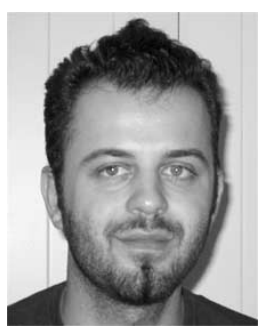

Alessio Giorgetti received the Laurea (cum laude) degree in telecommunication engineering from the University of Pisa, Italy, in 2002. He is currently working toward the Ph.D. degree at the "Center of Excellence for Communications Networks Engineering" of Scuola Superiore Sant'Anna di Studi Universitari e di Perfezionamento, Pisa, Italy.

His research interests include wavelength division multiplexing (WDM) networks design, fault tolerance in optical networks, and next generation synchronous optical network/synchronous digital hierarchy (SONET/SDH) technologies.

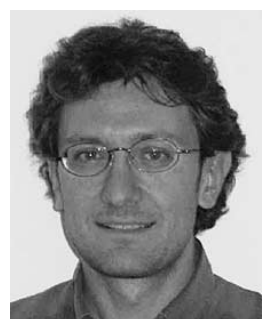

Luca Valcarenghi (S'00-M'02) received the Laurea degree in electronics engineering from Politecnico di Torino, Italy, in 1997, and the M.S. degree in electrical engineering and the Ph.D. degree in electrical engineering-telecommunications from the University of Texas at Dallas (UTD), in 1999 and 2001, respectively.

Between January 2002 and August 2002, he was a Research Associate of the Optical Networking Advanced Research (OpNeAR) Lab of the University of Texas at Dallas Erik Jonsson School of EE/CS. Since September 2002, he has been an Assistant Professor at the Scuola Superiore Sant'Anna di Studi Universitari e di Perfezionamento, Pisa, Italy. $\mathrm{He}$ has coauthored more than two dozen papers published in international journals and presented in leading international conferences. His main research interests are optical networks design, analysis, and optimization; artificial intelligence optimization techniques; communication networks reliability; Internet protocol (IP) over wavelength division multiplexing (WDM) networking; and quality of service (QoS) in network infrastructures for grid computing.

Dr. Valcarenghi has been part of the Organizing Committee and Technical Program Committee of international conferences such as Optical Networking and Systems Symposium at IEEE GLOBECOM 2003, and Optical Communication Networks and Systems Symposium at IEEE GLOBECOM 2004

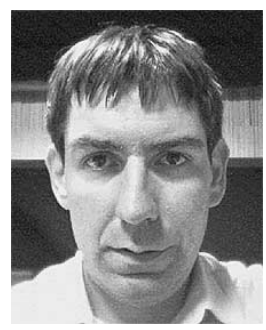

Piero Castoldi (S'93-A'96) received the Laurea (cum laude) degree in electrical engineering from the University of Bologna, Italy, in 1991, and the Ph.D. degree from the University of Parma, Italy, in 1996.

In 1996 and 1997, he was a Postdoctoral Researcher at the Department of Electrical Engineering, Princeton University, Princeton, NJ, where he was also hosted in the summers 1999 and 2000. From December 1997 to February 2001, he was an Assistant Professor at the Faculty of Engineering of the University of Parma, where he received the tenure. Since March 2001, he has been an Associate Professor in Telecommunications at the Scuola Superiore Sant'Anna di Studi Universitari e di Perfezionamento, Pisa, Italy, where he is responsible for the networks group. In January and February 2002, he was a Visiting Professor at the University of Texas at Dallas. He also collaborates in several projects of the Inter-University National Consortium for Telecommunications (CNIT) and since March 2003 has been the Director of the CNIT National Photonic Networks Laboratory in Pisa, Italy. He has taught several courses at the undergraduate and graduate level at the University of Parma and at Scuola Sant'Anna. The scientific activity has covered the area of digital transmission, telecommunication networks performance, and, more recently, provisioning, protection, and switching techniques for next generation optical networks. He is the author of more than 60 technical papers published in international conferences and journals and of an international book on code division multiple access (CDMA). 